\title{
Novel, low-cost alternative technologies to tackle practical, industrial conundrums - a case study of batteries
}

\author{
Victor K. Y. Chan ${ }^{1, a}$ and Sok Lei Leong ${ }^{1}$ \\ ${ }^{1}$ Macao Polytechnic Institute, School of Business, Rua de Luís Gonzaga Gomes, Macao, China.
}

\begin{abstract}
Whereas batteries in comparison with most other means of energy storage are more environmentally friendly and economical in their operation, they are beset by low energy replenishment rates, low energy storage density, high capital cost of themselves, and high capital cost of energy replenishment infrastructures. Mainly based on ergonomics, this paper proposes a novel, low-cost alternative technology to practically and industrially make these weaknesses irrelevant to some extent without calling for revolutionary technological breakthroughs in material science, batteries' microstructures, or battery manufacturing technologies. The technology takes advantage of modularization of battery systems, prioritization of charging and discharging of battery module(s) according to ease of unloading and/or loading the battery module(s) and/or ease of loading replacement battery module(s) of the battery module(s).
\end{abstract}

\section{Introduction}

Through discharging energy stored, charged batteries can power electrical and/or electronic instruments, equipment, devices, and/or machineries, which comprise and/or are connected to housings where such batteries are usually installed.

On the one hand, as particularly but not exclusively manifested by battery-powered electrical vehicles versus internal combustion vehicles, battery power is far more environmentally friendly [1] and economical in its operation in terms of the recurrent cost than most other means of energy storage [2]. The former is vulnerable to a range of vexed conundrums on the other hand. Such conundrums are ascribable to the weaknesses of battery power vis-à-vis other means of energy storage in respect of the former's fundamental and practical properties as elucidated below as well as the compromise between such properties:

1. Low energy replenishment rates: As far as energy replenishment through charging is concerned, the current, non-experimental battery charging technologies permit an energy delivery rate in kilowatts $(\mathrm{kW})$ [3] in contrast with, for example, thousands of $\mathrm{kW}$ in the case of a typical gas pump for replenishing energy stored in internal combustion vehicles. In fact, such rates are limited not only by the charging technologies but also the capacity of and compatibility with the local electric power grid [3]. Low charging rates result in prolonged charging durations.

2. Low energy storage density: Energy storage density refers to the energy stored per unit weight of the

\footnotetext{
${ }^{\text {a }}$ Corresponding author: vkychan@ipm.edu.mo
}

storage system, which is over ten times lower for batteries than gasoline, for example [3]. A consequence in the case of vehicles is electric vehicles' shorter vehicle ranges between energy replenishments than those of internal combustion vehicles [1,4]. Another concomitant consequence is the relatively large sizes and heavy weights of batteries themselves and their housings where the batteries are installed. Worst still, for the same type of batteries, their sizes and weights perceivably increase with their total energy storable. As such, especially but not exclusively, instruments, equipment, devices, and/or machineries consuming considerable power and/or energy necessitate batteries of commensurately considerable sizes and weights. Typical examples of instruments, equipment, devices, and/or machineries consuming considerable power and/or energy include electric vehicles, which usually comprise housings where battery systems are installed, so quite many aspects of electric vehicle design are usually sacrificed in order to accommodate sizable and heavy-weight batteries.

3. High capital cost of energy replenishment infrastructures: Such infrastructures usually involve not only standardized and inter-operable charging facilities but also upgrade of the local electric power grid [3] and thus may sometimes call for mega-projects at the national or even international level to implement. The odds are also that such implementation is exceptionally political and prone to obstinate resistance, especially, when 
standardization or other kinds of vested interests are concerned [3].

4. High capital cost of batteries: The higher capital cost of batteries than that of other means of energy storage render the overall capital cost of electrical and/or electronic instruments, equipment, devices, and/or machineries with batteries as the means of energy storage higher than that of comparable products adopting other means of energy storage [1, 3]. Such a difference is obvious when it comes to electric vehicles versus internal combustion vehicles $[1,3]$.

For the sake of tackling these four weaknesses, effort of current research in both academia and industry is highly focussed on material science and battery microstructures to discover and/or synthesize ameliorated battery chemistry and anode/cathode materials that in combination can give rise to higher charging rates and energy storage density and on inventing more ingenious manufacturing technologies to lower the production cost of batteries of a particular chemistry, anode/cathode materials, and battery microstructures [5]. Both nature scientists and social scientists have also researched into ways to construct charging infrastructures at minimal cost and incentivize investment in such infrastructures [3]. It is true that all such research has borne fruit $[3,5]$. Nonetheless, much less accentuation has been accorded to leveraging ergonomics of battery usage to make the four aforesaid weaknesses irrelevant to some extent, enabling even batteries based on current technologies of material science and battery microstructures to practically and industrially power a broader range of electrical and/or electronic instruments, equipment, devices, and/or machineries and thus replace other means of energy storage prevailing in such applications.

This paper proposes an ergonomics-based, novel, low-cost alternative technology, which is now a patent pending of the authors, to fill this gap. The paper further illustrates why and how such a technology may be able to practically and industrially make the four aforementioned weaknesses irrelevant to some extent and thus pave the way for batteries powering such electrical and/or electronic instruments, equipment, devices, and/or machineries as electrical vehicles without revolutionary technological breakthroughs in material science and battery microstructures and/or heavy investment in the invention of improved battery manufacturing technologies being a prerequisite for the technology's deployment in such real-life applications.

\section{The ergonomics of battery energy replenishment}

The four weaknesses of battery power as enumerated in the last section are manifested in the replenishment of energy stored in battery systems. There are two obvious approaches to replenish the energy stored in a partially or fully discharged battery system installed in a housing, namely,

1.charging (or recharging) the discharged battery system and
2. replacing the discharged battery system with a replacement battery system storing more energy than the discharged battery system whilst the discharged battery system is optionally to be charged (or recharged) with some charging facilities and to replace another discharged battery system subsequently.

In view of weakness 2 expounded in the last section, especially, if not exclusively, for aforementioned instruments, equipment, devices, and/or machineries consuming considerable power and/or energy, both these two approaches are practically beset by a fundamental difficulty to unload such battery systems of considerable sizes and weights from the housings and load (or reload) such battery systems after charging or load replacement battery systems, resulting in the following scenarios for the aforesaid approaches 1 and 2 .

In approach 1 , either the discharged battery systems must be charged when still remaining installed in the housings (i.e. in situ) or the discharged battery systems must be unloaded from the housings with appreciable effort before the discharged battery systems can be charged (or recharged) somewhere else with charging facilities and subsequently loaded (or reloaded) into the housings again with appreciable effort.

Charging the discharged battery systems when still remaining installed in the housings implies the necessary availability of charging facilities in the vicinity of the housings. For example, in the case of electric vehicles, this in turn implies immense infrastructures of charging facilities in a large number of parking spaces where the electric vehicles park and thus their discharged battery systems are optionally charged concurrently given that weakness 1 detailed in the last section confines charging to electric vehicles parking for protracted durations. SAE J1772 [6], IEC 62196 [7], and CHAdeMO [8] concern typical standards of such charging facilities. Weakness 3 as explained in the last section renders extensive availability of parking spaces with such immense infrastructures rather economically infeasible.

Alternatively, unloading discharged battery systems from their housings before charging and subsequent loading (or reloading) the charged battery systems implies the necessary availability of trained personnel and/or specialized loading and/or unloading facilities in the vicinity of the housings. For example, in the case of electric vehicles, this in turn implies immense infrastructures of trained personnel and/or specialized loading and/or unloading facilities in parking spaces or designated service stations where electric vehicles park or stop over and thus the electric vehicles' discharged battery systems are optionally unloaded for charging somewhere else and subsequently loaded (or reloaded) back into housings. Moreover, this also implies immense infrastructures to transport the discharged battery systems between such parking spaces or service stations and where there are charging facilities to charge the discharged battery systems. All these immense infrastructures aggravate weakness 3 delineated in the last section.

In approach 2, the discharged battery systems must be unloaded from the housings with appreciable effort, and 
the replacement battery systems must be loaded into the housings again with appreciable effort. For example, in the case of electric vehicles, this in turn calls for immense infrastructures of trained personnel and/or specialized loading and/or unloading facilities at designated service stations where discharged battery systems are unloaded and replacement battery systems loaded. This again exacerbates weakness 3 elaborated in the last section.

Worst still, as with most engineering optimizations where cost poses constraints on the optimization of and the compromise between system properties, the capital cost of batteries (to which weakness 4 of the last section relates) surges when attempting to increase charging rates (to which weakness 1 above relates) and energy storage density (to which weakness 2 above relates) [9]. In summary, both approaches 1 and 2 are plagued by some or all of the four weaknesses of the last section.

\section{The proposed technology}

The proposed technology aims at a solution to the aforementioned fundamental difficulty to unload and/or load battery systems, especially but not exclusively, those of considerable sizes and weights, based on the following principles:

1. Each battery system in a housing is modularized such that it is composed of a number of battery modules, each (or at least each of some of them) being individually unloadable from and loadable into the housing with substantially less effort than unloading and loading the battery system as a whole and each (or at least each of some of them) being optionally, individually chargeable and/or dischargeable.

2. Prioritization of battery module(s) to be charged and/or prioritization of battery module(s) to be discharged depends on ease of unloading and/or loading the battery module(s) to be charged and/or ease of loading replacement battery module(s) of the battery module(s) to be charged and/or depend on ease of unloading and/or loading the battery module(s) to be discharged and/or ease of loading replacement battery module(s) of the battery module(s) to be discharged. The ease of unloading and loading of particular battery module(s) may be due to, inter alia, the geographical location(s) of the battery module(s) in the housing.

3. Optionally, when the battery system is charged as a whole, additional charging priority is accorded to battery module(s) that are less easily unloadable and/or less easily loadable and/or battery module(s) of which replacement battery module(s) are less easily loadable vis-à-vis battery module(s) that are more easily unloadable and/or more easily loadable and/or battery module(s) of which replacement battery module(s) are more easily loadable. Highness and lowness of such charging priority are realized respectively by high and low relative intensity of electric current for charging where relative intensity of electric current for charging battery module(s) is the ratio of the intensity of electric current for charging the battery module(s) to the energy storage capacity of the battery module(s), and low relative intensity of electric current for charging may but does not necessarily mean not charging at all, i.e., zero intensity of electric current for charging.

4. Optionally, when the battery system is discharged, whether or not to power instrument(s), equipment, device(s), and/or machinery(ies), additional discharging priority is accorded to battery module(s) that are more easily unloadable and/or more easily loadable and/or battery module(s) of which replacement battery module(s) are more easily loadable vis-à-vis battery module(s) that are less easily unloadable and/or less easily loadable and/or battery module(s) of which replacement battery module(s) are less easily loadable. Highness and lowness of such discharging priority are realized respectively by high and low relative intensity of electric current for discharging where relative intensity of electric current for discharging battery module(s) is the ratio of the intensity of electric current for discharging the battery module(s) to the energy storage capacity of the battery module(s), and low relative intensity of electric current for discharging may but does not necessarily mean not discharging at all, i.e., zero intensity of electric current for discharging.

5. Optionally, battery module(s) that are more easily unloadable and/or more easily loadable and/or battery module(s) of which replacement battery module(s) are more easily loadable are accorded additional discharging priority for being discharged whereas battery module(s) that are less easily unloadable and/or less easily loadable and/or battery module(s) of which replacement battery module(s) are less easily loadable are accorded additional charging priority for being charged such that the battery module(s) being discharged charge(s) the battery module(s) being charged.

In essence, this proposed technology is to keep, to a possible extent, battery module(s) that are more easily unloadable and/or more easily loadable and/or battery module(s) of which replacement battery module(s) are more easily loadable at a lower state of charge than battery module(s) that are less easily unloadable and/or less easily loadable and/or battery module(s) of which replacement battery module(s) are less easily loadable. This way, battery module(s) that are more easily unloadable and/or more easily loadable and/or battery module(s) of which replacement battery module(s) are more easily loadable are more likely in more need of energy replenishment than battery module(s) that are less easily unloadable and/or less easily loadable and/or battery module(s) of which replacement battery module(s) are less easily loadable. To replenish the energy stored in the battery system, users can choose, inter alia, either one of the following options:

1.unloading battery module(s) that are more easily unloadable and/or more easily loadable, charging the unloaded battery modules(s), and loading (or reloading) the charged battery module(s) with less 
effort and less specialized facilities than analogously unloading, charging, and loading (or reloading) battery module(s) that are less easily unloadable and/or less easily loadable and with substantially less effort and less specialized facilities than analogously unloading, charging, and loading (or reloading) the battery system as a whole.

2.unloading battery module(s) that are more easily unloadable and/or battery module(s) of which replacement battery module(s) are more easily loadable and loading charged replacement battery module(s) with less effort and less specialized facilities than analogously unloading battery module(s) that are less easily unloadable and/or battery module(s) of which replacement battery module(s) are less easily loadable and loading charged replacement battery module(s) with substantially less effort and specialized facilities than unloading the battery system as a whole and loading a charged replacement battery system as a whole.

Both these options 1 and 2 concomitantly replenish the energy stored in battery module(s) more in need of stored energy replenishment.

For option 1, it is noteworthy that unloading, charging, and loading (or reloading) battery modules(s) that are more easily unloadable and/or more easily loadable need less specialized facilities than analogously unloading, charging, and loading (or reloading) battery module(s) that are less easily unloadable and/or less easily loadable because the former situation obviously involves less sophistication in disassembling, conveyance, assembling, etc. Likewise, unloading, charging, and loading (or reloading) battery modules(s) that are more easily unloadable and/or more easily loadable need substantially less specialized facilities than analogously unloading, charging, and loading (or reloading) the battery system as a whole because the former situation involves handling of smaller size(s) and weight(s) of battery module(s) than those of the battery system as a whole in the latter situation, the former situation involves smaller charging power than the latter situation, and the smaller size(s) and weight(s) of battery module(s) in the former situation allow minimal facilities to convey the battery module(s) between the housing and where charging facilities are located. For example, in the case of electric vehicles, unloading and loading (or reloading) battery modules(s) that are more easily unloadable and/or more easily loadable and conveyance of such unloaded battery module(s) between where the electric vehicles are parked and where charging facilities are located may be practically be performed manually by the users without specialized facilities whatsoever whilst charging may be performed with domestic and mainsbased charging facilities or the like at homes, offices, etc.

For option 2, unloading battery module(s) that are more easily unloadable and/or battery module(s) of which replacement battery module(s) are more easily loadable and loading charged replacement battery module(s) need less specialized facilities than analogously unloading battery module(s) that are less easily unloadable and/or battery module(s) of which replacement battery module(s) are less easily loadable and loading charged replacement battery module(s) because the former situation obviously involves less sophistication in disassembling, conveyance, assembling, etc. Likewise, unloading battery module(s) that are more easily unloadable and/or battery module(s) of which replacement battery module(s) are more easily loadable and loading charged replacement battery module(s) need substantially less specialized facilities than unloading the battery system as a whole and loading a replacement battery system as a whole because the former situation involves handling of smaller size(s) and weight(s) of battery module(s) than those of the battery system as a whole in the latter situation. Also, for this option 2, charging may be performed with domestic and mainsbased charging facilities or the like at homes, offices, etc.

In short, both options 1 and 2 reduce the use of specialized facilities and thus the cost of energy replenishment infrastructures (to which weakness 3 above relates). Better still, charging rates (to which weakness 1 above relates) are rendered by either of these two options somewhat irrelevant to the users' convenience and operational cost in that these two options' charging may be performed with domestic and mains-based charging facilities or the like at homes, offices, etc., obviating employment of specialized charging facilities, which are presumably expensive and relatively less readily accessible, for extended time periods. Likewise, with either options 1 or 2, although low energy storage density (to which weakness 2 above relates) of battery systems still leads to their considerable sizes and weights, the use of expensive, specialized charging facilities to handle large battery sizes and weights is no longer necessitated thanks to the proposed technology's modularization of battery systems and thus their energy replenishment. As a final result, with the proposed technology, there may be room for reducing the capital cost of batteries (to which weakness 4 above relates) given the less need to increase the charging rates (to which weakness 1 above relates) and the energy storage density (to which weakness 2 above relates), relaxing quite some capital cost optimization constraints.

\section{Conclusion}

Mainly based on the ergonomics of battery energy replenishment, this paper proposes a novel, low-cost alternative technology that may be able to practically and industrially make irrelevant to some extent the four major weaknesses of battery power vis-à-vis other means of energy storage and thus pave the way for batteries powering such electrical and/or electronic instruments, equipment, devices, and/or machineries as electrical vehicle without revolutionary technological breakthroughs in material science and battery microstructures and/or heavy investment in the invention of improved battery manufacturing technologies being a prerequisite for the technology's deployment in such reallife applications. 
The proposed technology takes advantage of modularization of battery systems, prioritization of charging and discharging of battery module(s) according to ease of unloading and/or loading the battery module(s) and/or ease of loading replacement battery module(s) of the battery module(s). This proposed technology may be able to eradicate the need for specialized charging facilities altogether and thus is particularly fit for densely populated metropolitans where space for such facilities (for example, private garages in the case of electric vehicle battery systems) is not only hardly available but also costly.

\section{Acknowledgments}

This article was supported by Grant (004/2013/A) of the Science and Technology Development Fund, the Government of Macao Special Administrative Region.

\section{References}

1. M. Hülsmann, D. Fornahl (eds.), Evolutionary Paths Towards the Mobility Patterns of the Future, Lecture Notes in Mobility (2014)

2. C.-S. N. Shiau, C. Samaras, R. Hauffe, J.J. Michalek, Impact of battery weight and charging patterns on the economic and environmental benefits of plug-in hybrid vehicles, 2009 Annual Meeting of the Transportation Research Board (2009)

3. M. Ralston, N. Nigro, Plug-In Electric Vehicles: Literature Review (2011)

4. L. Raykin, H.L. MacLean, M.J. Roorda, Implications of driving patterns on well-to-wheel performance of plug-in hybrid electric vehicles, Environ. Sci. Technol. 46, 6363-6370 (2012)

5. M.-C. Lin, M. Gong, B. Lu, Y. Wu, D.-Y. Wang, M. Guan, M. Angell, C. Chen, J. Yang, B.-J. Hwang, H. Dai, An ultrafast rechargeable aluminium-ion battery, Nature 520, 324-328 (2015)

6. SAE International, J1772 201210: SAE Electric Vehicle and Plug in Hybrid Electric Vehicle Conductive Charge Coupler (2012)

7. International Electrotechnical Commission, IEC 62196-3:2014: Plugs, Socket-Outlets, Vehicle Connectors and Vehicle Inlets - Conductive Charging of Electric Vehicles (2014)

8. CHAdeMO, Technological details, http://www.chademo.com/wp/technology/details/ (Accessed 14/01/2016)

9. Texas Instruments Incorporated, Characteristics of Rechargeable Batteries (2011) 